\title{
High prevalence of tuberculosis among adults with fever admitted at a tertiary hospital in north-western Tanzania
}

\author{
ALFRED J. MEREMO ${ }^{1}$, BENSON R. KIDENYA ${ }^{2}$, STEPHEN E. MSHANA ${ }^{3 *}$, RODRICK KABANGILA ${ }^{1}$ and \\ JOHANNES B. KATARAIHYA ${ }^{1}$ \\ ${ }^{1}$ Department of Internal Medicine, Weill Bugando School of Medicine, Catholic University of Health and Allied \\ Sciences-Bugando, Mwanza, Tanzania \\ ${ }^{2}$ Department of Biochemistry and Molecular Biology, Weill School of Medicine, Catholic University of Health and \\ Allied Sciences-Bugando, Mwanza, Tanzania \\ ${ }^{3}$ Department of Microbiology/Immunology Weill School of Medicine, Catholic University of Health and Allied \\ Sciences-Bugando, Mwanza, Tanzania
}

\begin{abstract}
Tuberculosis is a leading cause of death in developing countries where HIV is endemic. This hospital based study was done to estimate the magnitude of pulmonary and extra-pulmonary tuberculosis and to determine predictors of tuberculosis among febrile adults admitted at Bugando Medical Centre (BMC), Mwanza, Tanzania. A total of 346 adults febrile patients admitted in medical wards were studied. Sputum for AFB microscopy and chest X-rays was used to diagnose tuberculosis. Clinical features were collected using standardized data collection tool. HIV testing and CD4 counts were determined. Data were analyzed using STATA version 11 software. Of 346 febrile adults patients 116 $(33.5 \%)$ were diagnosed to have tuberculosis; of which $79(68.1 \%)$ and $37(31.9 \%)$ had pulmonary tuberculosis (PTB) and extra-pulmonary tuberculosis, respectively. Smear negative PTB were more common in HIV positive than in HIV negative patients ( $50 \%$ vs. 18.5\%, $\mathrm{p}=0.007)$. Extra-pulmonary tuberculosis was more common in HIV positive patients than pulmonary tuberculosis (86.4\% vs. $13.6 \%$ ), $\mathrm{p}=0.0001$ ). On multivariate logistic regression analysis the predictors of tuberculosis were; age above 35 years $(\mathrm{OR}=2.38, \mathrm{p}=0.007)$, cardinal symptoms $(\mathrm{OR}=37, \mathrm{p}<0.0001)$, pleural effusion $(\mathrm{OR}=24, \mathrm{p}=0.0001)$, and HIV status (OR $=3.2, \mathrm{p}=0.0001)$. Of 79 patients with PTB, $48(60.7 \%)$ were AFB smear positive and 31 (39.3\%) were AFB smear negative. HIV patients with smear negative tuberculosis had significantly lower CD4 count than HIV patients with smear positive tuberculosis ( 63.5 cells/ $\mu$ l versus 111.5 cells/ $\mu \mathrm{l}$ ) [MannWhitney test $\mathrm{p}=0.0431]$. No different in mortality was observed between patients with TB and those without TB admitted in BMC medical wards (28.5\% vs. $23.0 \%, \mathrm{p}=0.1318)$. Tuberculosis is the commonest cause of fever among adults patients admitted at BMC and is predicted by age above 35 years, positive HIV status, cardinal PTB symptoms, and pleural effusion. Routinely TB screening is highly recommended among adults with fever, cough, night sweating and wasting in countries where HIV is endemic.
\end{abstract}

Keywords: Tuberculosis, HIV/AIDS, fever, adults, Tanzania

\section{Introduction}

More than 11 million adults living with HIV/AIDS are also infected with tuberculosis (WHO 2007). In some sub-Saharan countries, one-third of patients with tuberculosis (TB) have HIV (WHO 2007). TB affects more than half of those infected with HIV (WHO 2007). In 2009 a total of 60,060 new TB cases (AFB+, AFB- and extrapulmonary) were reported in Tanzania. Out of

\footnotetext{
${ }^{*}$ Correspondence: Stephen E. Mshana; E-mail mshana72@yahoo.com
} 
these new cases $41.5 \%$ were smear positive. Of the total new TB cases $88 \%$ had known HIV status and $37 \%$ were found to be HIV positive (NTLP, 2009). The overlapping between tuberculosis and HIV epidemics has had a devastating impact on morbidity, mortality, and control, rendering tuberculosis the leading killer of HIV-positive people. More than $29 \%$ of global burden of TB is in Africa with 34\% related deaths (WHO 2007). TB in adults causes fever, often lasting weeks, retrosternal discomfort or pain due to mediastinal lymph node enlargement and pleuritic pain (Bruchfeld et al 2003). Symptoms vary from a gradual insidious onset with anorexia, weight loss, fatigue and low-grade fever to acute onset with fever, night sweats, productive cough and dyspnoea (Nyirenda et al 2006). In Malawi among adults patient admitted with fever mycobacterium was the commonest isolate from the blood (Lewis et al 2003). Other study in Malawi found the prevalence of Mycobacterium tuberculosis blood stream infection in HIV endemic areas to range from 9-12\% (Archibald et al 2000). In Tanzania 20.2\% of HIV adult patients were found to be infected with TB compared to $1.3 \%$ of HIV negative (Lennox et al 1998). Diagnosis of TB in developing countries where HIV is endemic brings more challenges (Shea et al., 2009).

Little is known about the magnitude of TB among febrile adults admitted in hospitals in Tanzania. This cross-sectional prospective study was conducted to determine the magnitude, predictors and case fatality rate of tuberculosis among febrile adults admitted at tertiary hospital in Northern-Western Tanzania.

\section{Materials and Methods}

\section{Study design and sample size}

A prospective cross-sectional hospital based study was conducted at Bugando Medical Centre (BMC) in Mwanza region in north-western Tanzania from June 2011 to December 2011. BMC is a tertiary hospital and University teaching hospital serving approximately 13 million people in the North-Western zone of Tanzania.

Sample size was estimated using Kish and Leslie formula (Kish, 1965) the proportion of $20 \%$ (Lennox et al 1998) was used in the formula; this is the prevalence of TB among HIV patients in Tanzania.

\section{Patients' recruitment and laboratory procedures}

All adults patients above 18 years with fever admitted in medical wards were enrolled into the study. Patients were asked to sign informed consent and were counselled for HIV testing. Sputum for acid fast bacilli (AFB) and blood for HIV testing and CD4 estimation were obtained as previously described (WHO 1998; Enarson et al., 2000; IUATLD 2000; Bakari et al 2008).

Patient clinical history and physical examination findings were collected using standardized data collection tool. Chest X-ray was done in all patients suspected to have tuberculosis. HIV testing was done as per Tanzania National algorithm and CD4+ counts determined using FACS Calibur (BD UK). 


\section{Tuberculosis diagnosis}

Smear positive tuberculosis was diagnosed if a patient had positive spot and early morning AFB smear or one positive smear with positive chest-X-ray findings (Bakari et al., 2008). Smear negative tuberculosis were diagnosed if a patient had three negative smear (spot, early morning, spot), and failed to respond on 14 days antibiotic treatment, but improved when antiTB is started. Extra pulmonary tuberculosis was defined as previously described (http://www.stoptb.org/wg/gli/assets/documents/TBcasedefinitions 20110506b.pdf).

\section{Quality control}

Adequate mucoid or mucopurulent sputum specimens were collected from TB suspects. The sputum specimens were transported immediately to the BMC laboratory and examined for the presence of AFB by experienced laboratory technician based on the standard operative procedures (Kubi \& Kent, 1985). All the slides were checked again by the three investigators who are expert in the subject and no discrepancy was observed. All chest -x-ray were read by experienced radiologist in the hospital. All patients were seen after 2 weeks and assessed for clinical improvement.

\section{Data analysis}

Data collected were entered into a computer using Epidata version 3.1 (CDC, Atlanta, USA) and analyzed using STATA version 11 (College Station, Texas, USA). Data were summarized in form of proportions and frequent tables for categorical variables. To assess the significance of their distribution chi-square test was used. For non parametric continuous variables, we used medians to summarize them and Mann-Whitney test to test for the significance of the difference of the medians between two groups. To determine predictors of tuberculosis, logistic regression analysis was performed, starting with univariate then followed by multivariate logistic regression analysis with the dichotomous outcome being tuberculosis or no tuberculosis.

Odds ratio with 95\% confidence interval was calculated to test for strength of association between predictor variables and tuberculosis. Predictors with p-value less than 0.05on univariate analysis were then fitted into the multivariate regression analysis and predictors with a p-value of less than 0.05 were considered to have significant strength of association.

In the present study cough, night sweating and wasting were considered as cardinal symptoms of tuberculosis and were combined together and tested if they independently predict tuberculosis in multivariate analysis. We further analyzed the strength of HIV on predicting the two types of tuberculosis. We used multinomial logistic regression with the outcome being no tuberculosis, pulmonary tuberculosis and extra pulmonary tuberculosis. Based on patients with no tuberculosis as the reference group, relative risk ratio with $95 \%$ confidence interval was calculated to test for strength of association between HIV and tuberculosis type.

\section{Ethical consideration}

Ethical clearance and approval was obtained from a joint Catholic University of Health and Allied Sciences and Bugando Medical Centre Research and Publications Ethics Review Board. Written informed consent was obtained from each patient. If patient was unable to provide consent, written consent was obtained from their legal guardians. 


\section{Results}

\section{Demographic and other characteristics}

Over the study period 1,552 patients were admitted to the medical wards at BMC and were screened for eligibility. A total of 417 (26.9\%) met inclusion criteria of whom 346 (83\%) were enrolled into the study. The median age was 35 (inter quartile range [IQR] 25-48) years and 179 $(51.7 \%)$ were male. The median temperature was 38.5 with IQR of $38-39{ }^{\circ} \mathrm{C}$. A total of 156 (45.1\%) of the participants had HIV infection; of these $112(71.8 \%)$ had WHO stage four. The median CD4 count among patients with HIV infection was 78 cells/ $\mu \mathrm{l}$.

\section{Magnitude and predictors of Tuberculosis}

Of 346 adult febrile patients 116 (33.5\%) were confirmed to have tuberculosis; of which 79 $(68.1 \%)$ and $37(31.9 \%)$ had pulmonary tuberculosis and extra-pulmonary tuberculosis respectively. Of 79 patients with PTB, 48 (60.7\%) were AFB smear positive and 31 (39.3\%) were AFB smear negative. All 37 patients with extra-pulmonary tuberculosis had pleural effusion.

Table 1: Univariate analysis of predictors of TB among febrile adult patients

\begin{tabular}{|c|c|c|c|c|c|}
\hline Variable (N) & Response & Positive TB $(\mathrm{N}=116)$ & Odd ratio & $95 \% \mathrm{CI}$ & P value \\
\hline \multirow[t]{2}{*}{ Age } & $\leq 35$ year $(179)$ & & & & \\
\hline & >35 years (167) & $71(42.5 \%)$ & 2.2 & $1.395-3.475$ & 0.001 \\
\hline \multirow[t]{2}{*}{ Sex } & Male ( 179) & $59(32.9 \%)$ & 1 & & \\
\hline & Female (167) & $57(34.1 \%)$ & 1.05 & $0.674-1.64$ & 0.818 \\
\hline \multirow[t]{2}{*}{ Chronic Cough } & Absent (154) & $1(0.65 \%)$ & 1 & & \\
\hline & Present (192) & $115(59.9 \%)$ & 228 & $31.3-1667$ & $<0.0001$ \\
\hline \multirow[t]{2}{*}{ Night sweat } & Absent (299) & $81(27.0 \%)$ & 1 & & \\
\hline & Present (47) & $35(74.5 \%)$ & 7.8 & $3.88-15.86$ & $<0.0001$ \\
\hline \multirow[t]{2}{*}{ Wasting } & Absent(254) & $56(22.0 \%)$ & 1 & & \\
\hline & Present(92) & $60(65.2 \%)$ & 6.6 & $3.93-11.16$ & $<0.0001$ \\
\hline \multirow[t]{2}{*}{ Pleural effusion } & Absent (289) & $65(22.5 \%)$ & 1 & & \\
\hline & Present (57) & $51(89.5 \%)$ & 29.3 & $12-71.31$ & $<0.0001$ \\
\hline \multirow[t]{2}{*}{ HIV status } & Negative(190) & $32(16.8 \%)$ & 1 & & \\
\hline & Positive(156) & $84(53.9 \%)$ & 5.8 & $3.5-9.43$ & $<0.0001$ \\
\hline \multirow[t]{3}{*}{$W B C$} & $4000-11000(123)$ & $42(34.2 \%)$ & 1 & & \\
\hline & $\leq 4000 / \mu \mathrm{l}(74$ & $39(52.7 \%)$ & 2.14 & $1.19-3.87$ & 0.011 \\
\hline & $>4000 / \mu \mathrm{l}(149)$ & $35(23.5 \%)$ & 0.59 & $0.34-1.00$ & 0.053 \\
\hline \multirow[t]{2}{*}{ Anaemia } & $\geq 10 \mathrm{~g} / \mathrm{dl}(109)$ & $24(22.0 \%)$ & 1 & & \\
\hline & $<10 \mathrm{~g} / \mathrm{dl}(237)$ & $92(38.8 \%)$ & 2.24 & $1.33-3.79$ & 0.002 \\
\hline
\end{tabular}

WBC $=$ white blood cells

On univariate logistic regression analysis night sweat, cough, wasting, positive HIV status, pleural effusion and anaemia were found to predict tuberculosis (Table 1). On multivariate logistic regression analysis the predictors of tuberculosis were; age above 35 years $(\mathrm{OR}=2.38$, $\mathrm{p}=0.007)$, cardinal symptoms $(\mathrm{OR}=37, \mathrm{p}=<0.0001)$, pleural effusion $(\mathrm{OR}=24, \mathrm{p}=0.0001)$, and HIV status $(\mathrm{OR}=3.2, \mathrm{p}=0.0001)$ (Table 2). 
Table 2: Multivariate logistic regression analysis of predictors of tuberculosis among febrile adults

\begin{tabular}{llll}
\hline Variable & Odd ratio & $\mathbf{9 5 \%}$ CI & P value \\
\hline Age $>$ 35 years & 2.38377 & $1.2658-4.489$ & 0.007 \\
Sex & 1.49688 & $0.8011-2.796$ & 0.206 \\
Cardinal symptoms & 37.3454 & $7.695-181.243$ & $<0.0001$ \\
Pleural effusion & 24.35281 & $9.297-63.789$ & $<0.0001$ \\
HIV status & 3.2313 & $1.676-6.2289$ & $<0.0001$ \\
Anaemia & 0.9612 & $0.4692-1.9693$ & 0.914 \\
\hline
\end{tabular}

\section{HIV status and tuberculosis}

Of 48 patients with smear positive TB, 22 (45.8\%) were HIV positive and 26 (54.2\%) were HIV negative. Smear negative PTB were more common in HIV positive than in HIV negative patients $(83.9 \%$ vs. $16.1 \%, \mathrm{p}=0.007)$ (Table 3$)$. When compared to patients without tuberculosis as a reference group, HIV patients were much more likely to have extra pulmonary tuberculosis (RRR 14.0 [95\% CI 5.3 - 37.5], p<0.001) than having pulmonary tuberculosis (RRR 4.2 [95\% CI 2.5 - 7.3], p<0.001) (Figure 1). Though HIV predicts tuberculosis, HIV positive serostatus predicts more extra-pulmonary tuberculosis than it does for pulmonary tuberculosis.

The median CD4 counts of HIV patients with smear positive tuberculosis was 111.5 cells/ $\mu \mathrm{l}$ compared to 63.5cells/ $\mu \mathrm{l}$ of HIV patients with smear negative tuberculosis (MannWhitney test $\mathrm{p}=0.0431$ ). HIV patients with smear negative tuberculosis had significantly lower CD4 count than HIV patients with smear positive tuberculosis.

Table 3: HIV status in relation to smear positive and smear negative pulmonary tuberculosis

\begin{tabular}{llll}
\hline Variable & \multicolumn{2}{l}{ Pulmonary Tuberculosis } & Total \\
\hline HIV status & Smear positive & Smear negative & \\
Negative & $22(81.5 \%)$ & $5(18.5 \%)$ & 27 \\
Positive & $26(50.0 \%)$ & $26(50.0 \%)$ & 52 \\
Total & $\mathbf{4 8}$ & $\mathbf{3 1}$ & $\mathbf{7 9}$ \\
\hline
\end{tabular}

Chi square $7.3876, \mathrm{p}=0.007$

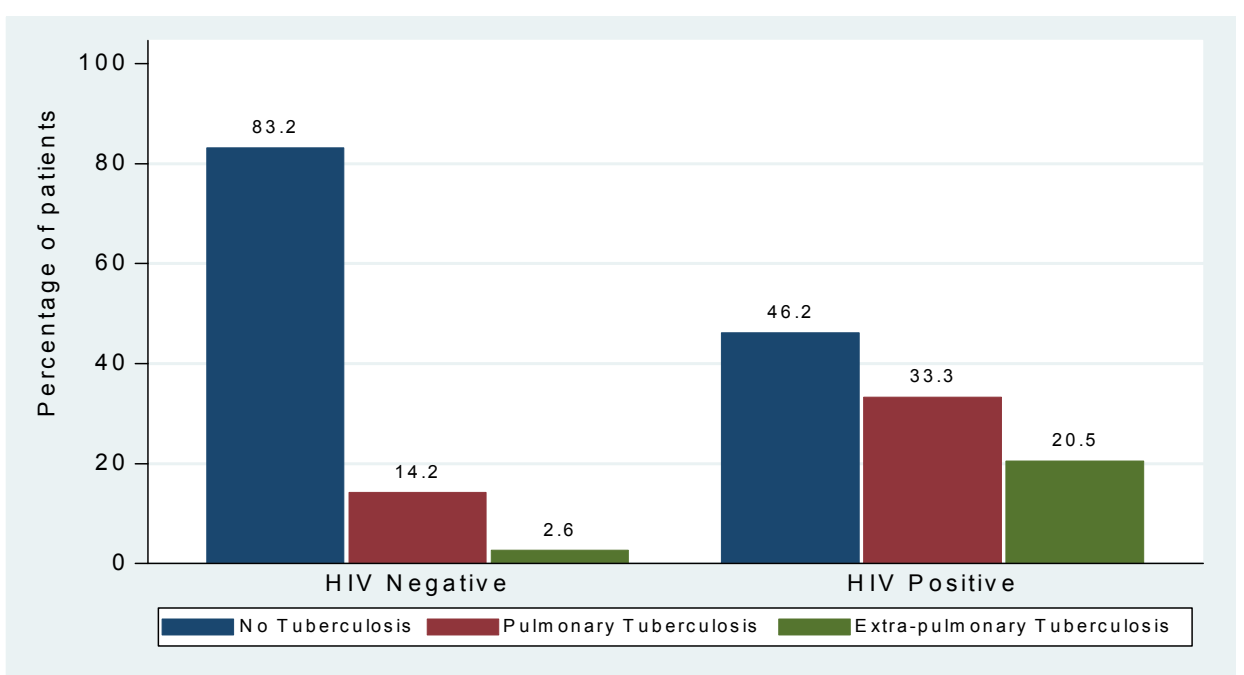

Figure 1: HIV status in relation to type of tuberculosis 
HIV positive serostatus predicts more extra-pulmonary tuberculosis than it does for pulmonary tuberculosis. RRR 4.2 [95\% CI 2.5 - 7.3] versus 14.0 [95\% CI 5.3 - 37.5] all differences observed were statistically significant $\mathrm{p}$-value $<0.001$.

\section{Discussion}

In this study, we have confirmed the high prevalence of tuberculosis in North-western Tanzania among HIV febrile adults. Overall the prevalence of tuberculosis observed in this study agrees with that of other studies in Africa (Corbett et al., 2003; Dagnara et al., 2011). Among TB patients $72 \%$ were co-infected with HIV. There have been several studies of the prevalence of HIV in TB patients in different parts of Africa ranges from $6.1 \%$ in Nigeria to $57.1 \%$ in Ethiopia (Anteyi $e t$ al., 1996; Bruchfeld et al., 2002). The prevalence of HIV in TB patients observed in this study is higher than $57.1 \%$ reported previously in the same area (Range et al., 2007) and National TB and HIV co-infection of $37 \%$ (NTLP, 2009); this could be explained by the fact that this was a tertiary hospital based study.

Majority of HIV patients in our study were in WHO stage 4, this could be contributed by poor health seeking behaviour in developing countries. As reported previously patients with smear positive tuberculosis had higher median CD4+ count than HIV patients with smear negative tuberculosis (Rawat et al., 2008; Sameer et al., 2011; Mbwana et al., 2011). HIV infected smear positive patient tend to secrete significant fewer organisms per $\mathrm{ml}$ than HIV negative patient (Long et al., 1991). Our study has confirmed that smear negativity increases as immunosuppression increases (Shea et al 2009; Sameer et al., 2011).

Various risk factors have been assessed in relation to tuberculosis in present study. Patient's risk factors associated with increased risk of tuberculosis includes chronic cough, wasting, advanced age, HIV status, Diabetes Mellitus, Malnutrition etc (Lewis et al 2003, David et al 2004). In the present study as in others studies (Bruchfeld et al., 2003; Mtei et al., 2005) factors found to predict tuberculosis on univariate logistic regression analysis were age above 35 years, cough more than 2 weeks, night sweats, severe wasting, present of pleural effusion, positive HIV status and anaemia. Independent risk factors found to predict tuberculosis on multivariate logistic regression analysis were cardinal symptoms of tuberculosis (chronic cough, night sweating and wasting); the risk of tuberculosis in patients with these three symptoms was more than 10 times compared to patient without these symptoms. Other factors found to predict tuberculosis on multivariate analysis were positive HIV status, presence of pleural effusion and age above 35 years. Clinicians should know these factors so that they can have high index of suspicion of tuberculosis diagnosis in patients presenting with these factors. In the present study HIV has been a statistically significant predict extra-pulmonary tuberculosis than it does for pulmonary tuberculosis. Previous studies have reported that extrapulmonary disease is more common in HIV infected patients (Harries et al., 1998).

The case fatality rate observed was $28.4 \%$; this seems to be higher than previously reported in Tanzania (van den Broek et al., 1998; Mtei et al 2005; Range et al., 2007). This could be explained by the fact that majority of our patients with tuberculosis were HIV infected and WHO stage 4 with the evidence of severe immunosuppression based on CD4+ counts (Kiertiburanakul et al., 2012). 
The magnitude of tuberculosis in this cohort might be low due to the limitation of this study which was failure to perform sputum and blood culture for Mycobacterium tuberculosis complex spp.

In conclusion, sputum smear is still an important diagnostic tool among HIV patients with CD4 count more than 111.5cells/ $\mu$ l. Other techniques like routine culture, GeneXpert are urgently needed in our setting so that TB control can be assured and sustained. Strategies such as creating public awareness and the involvement of the private health sector, traditional practitioners and religious institutions in HIV and TB control should be emphasized in order to improve health seeking behaviour among HIV patients in developing countries.

\section{Acknowledgements}

The authors would like to acknowledge the technical support provided by Zacharia Igembe, Lisa Gerwing and John Muyombe. We thank member of Department of Internal Medicine of Bugando Medical Centre for their excellent technical assistance. This study received financial support from College of Health and Allied Sciences of the University of Dodoma, Tanzania.

\section{References}

Anteyi, E.A., Idoko, J.A., Ukoli, C.O., Bello, C.S. (1996) Clinical pattern of human immunodeficiency virus infection (HIV) in pulmonary tuberculosis patients in Jos, Nigeria. Africa Journal of Medical Sciences 25, 317-21.

Bakari, M., Arbeit, R.D., Mtei, L., Lyimo, J., Waddell, R., Matee, M., Cole, B.F., Tvaroha, S., Horsburgh, C.R, Soini, H., Pallangyo, K., von Reyn, C.F.(2008) Basis for treatment of tuberculosis among HIV-infected patients in Tanzania: the role of chest $x$-ray and sputum culture. BMC Infectious Diseases 6, 8:32.

Bruchfeld, J., Aderaye, G., Palme, I.B., Bjorvatn, B., Britton, S., Feleke, Y., Källenius, G., Lindquist, L. (2003) Evaluation of outpatients with suspected pulmonary tuberculosis in a high HIV prevalence setting in Ethiopia: clinical, diagnostic and epidemiological characteristics. Scandnavian Journal of Infectious Diseases 34, 331-337.

Bwana, V., Tenu, F., Magesa, S.M., and Mfinanga, A.G. (2011) Smear positive pulmonary tuberculosis among HIV patients receiving highly active antiretroviral therapy in Dar es Salaam, Tanzania. Tanzania Journal of Health Research, 13:1.

Corbett, E.L., Watt, C.J., Walker, N., Maher, D., Williams, B.G., Raviglione, M.C., Dye, C. (2003) The growing burden of tuberculosis: Global trends and interaction with the HIV epidemic. Archive of Internal Medicine 163, 1009-1021.

Dagnara, A.Y., Adjok, K., Tchaptchet, H.S, Patassi, A.A., Sadzo Hetsu, D., Awokou, F., Tidjani, O. (2011) Prevalence of HIV-TB co-infection and impact of HIV infection on pulmonary tuberculosis outcome in Togo. Bulletin de la Societe de Pathologie Exotique 104, 342-346.

David, R.M., Christopher, W.W., Mark D.Z., Peter M.D., Ram H B., Andrew J.K., Robert M.S., Buddha B., Lennox, K.A. \& Reller, 1.B. (2004) The etiology of febrile illness in adults presenting to Patan Hospital in Kathmandu, Nepal. American Journal of Tropical Medicine and Hygiene 70, 670-675. 
Harries A.D. (1998) Tuberculosis in HIV-infected persons with special emphasis on sub-Saharan Africa. Journal of Infection 37, 205-209.

International Union against Tuberculosis and Lung Disease: Sputum examination for tuberculosis by direct microscopy in low income countries. 5th edition. Paris, France: IUATLD; 2000.

Kent, P.T. \& Kubica, G.P. (1985) Public Health Mycobacteriology. A Guide for the Level III Laboratory. U.S. Department of Health and Human Services. Atlanta, Georgia, 207 pp.

Kiertiburanakul, S., Watcharatipagorn, S., Chongtrakool, P. \& Santanirand, P. (2012) Epidemiology of bloodstream infections and predictive factors of mortality among HIVinfected adult patients in Thailand in the era of highly active antiretroviral therapy. Japanese Journal of Infectious Diseases 65, 28-32

Kim, L., Heilig, C.M., McCarthy, K.D., Phanuphak, N., Chheng, P., Nong, K., Quy, H.T., Sar, B., Cain, K.P. \& Varma, J.K. (2012) Symptom screen for identification of highly infectious tuberculosis in people living with HIV in Southeast Asia. Journal of Acquired Immune Deficiency Syndrome. doi: 10.1097/QAI.0b013e318256b3db

Kish, L. (1965) Survey Sampling New York: Wiley Interscience Publication .

Lan, R., Yang, C., Lan, L., Ou, J., Qiao, K., Liu, F. \& Gao, Q. (2011) Mycobacterium tuberculosis and non-tuberculous mycobacteria isolates from HIV-infected patients in Guangxi, China. International Journal of Tuberculosis and Lung Diseases 15, 1669-1675.

Lennox, K., Archibald, M.O., Pallangyo, K.J. \& Reller, L.B. (1998) Fatal Mycobacterium tuberculosis Bloodstream Infections in Febrile Hospitalized Adults in Dar es Salaam, Tanzania. Clinical Infectious Diseases 26, 290-296.

Lewis, D.K, Peter, R.P.H., Schijffelen, M.J., Joaki, G.R.F, Walsh, A.L. \& Kublin, J.K (2003). Clinical indicators of mycobacteraemia in adult admitted to hospital in Blantrye. Malawi Medical Journal 15, 56-60.

Long, R., Scalcin, M., Manfrea, L., Baptiste, M.J. \& Hershfiel, E. (1991) The impact of HIV on the usefulness of sputum smears for the diagnosis of TB. American Journal of Public Health 81, 1326-1328.

Mtei, L., Matee, M., Herfort, O., Bakari, M., Horsburgh, C.R, Waddell, R., Cole, B., Kreiswirth, B., Pallangyo, K. \& von Re, C.F. (2005) High rates of clinical and subclinical tuberculosis among HIV-infected ambulatory subjects in Tanzania. Clinical Infectious Diseases 40, 1500-1507.

NTLP (2009) National Tuberculosis and Leprosy Programme Annual Report 2009. Ministry of Health and Social Welfare, Dar es Salaam, Tanzania

Nyirenda, T. (2006) Epidemiology of Tuberculosis in Malawi. Malawi Medical Journal 18, 147159.

Range, N., Magnussen, P., Mugomela, A., Malenganisho, W., Changalucha, J., Temu, M.M., Mngara, J., Krarup, H., Friis, H. \& Andersen, A.B. (2007) HIV and parasitic coinfections in tuberculosis patients: a cross-sectional study in Mwanza, Tanzania. Annals of Tropical Medicine and Parasitology 101, 343-351.

Rawat, J., Sindhwani, G. \& Juya, R. (2008) Clinico-radiological profile of new smear positive pulmonary tuberculosis cases among young adult and elderly people in tertiary care hospital at Deheradun (Uttarakhad). Indian Journal of Tuberculosis 55, 84-90. 
Shea, Y.R., Davis, J.L., Huang, L., Kovacs, J.A., Masur, H., Mulindwa, F., Opus, S., Chow, Y. \& Murray, P.R. (2009) High sensitivity and specificity of acid-fast microscopy for diagnosis of pulmonary tuberculosis in an African population with a high prevalence of human immunodeficiency virus. Journal of Clinical Microbiology 47, 1553-1555.

Singhal, S., Mahajan, S.N., Diwan, S.K, Gaidhane, A. \& Quazi, Z.S. (2011) Correlation of sputum smear status with CD4 count in cases of pulmonary tuberculosis and HIV co-infected patients -A hospital based study in rural area of Central India. Indian Journal of Tuberculosis 58, 108-112.

van den Broek, J., Mfinanga, S., Moshiro, C., O'Brien, R., Mugomela, A. \& Lefi, M. (1998) Impact of human immunodeficiency virus infection on the outcome of treatment and survival of tuberculosis patients in Mwanza, Tanzania. International Journal of Tuberculosis Lung Disease 2, 547-552.

WHO (2007) WHO Report 2007: Global tuberculosis control: surveillance, planning, Financing. Geneva: World Health Organization, 2007. (WHO/HTM/TB/2007.376.)

WHO (1998) Laboratory services in tuberculosis control: Part II Microscopy 